\title{
La educación del talento académico en los estudiantes desde la física
}

DOI: https://doi.org/10.33262/ap.v2i2.28

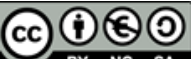

The education of the academic talent of students since physics

Ariel León Arencibia., ${ }^{1}$ Beatriz de la Caridad Dorvigny González. ${ }^{2}$ \& Bárbaro Evelio Rodríguez Brito. ${ }^{3}$

\begin{abstract}
.
This word reveals as problematic the insufficient preparation of pre-university teachers to assume, among their objectives, the education of students' academic talent since Physics as a subject. Instruments are presented to the teacher for their preparation in the education of the academic talent of pre-university students since Physics, from new semiotic foundations that allow to use the cultural and daily codes to intrinsically motivate the students, principles are proposed, definitions in that this is sustained, as well as its conditioning elements, dimensions and indicators, which are specified through the guidelines, with methodological steps.
\end{abstract}

Keywords: Education, Academic Talent, Physics as a subject, teacher preparation.

\section{Resumen.}

En este trabajo se revela como problemática la insuficiente preparación de los docentes para asumir, entre sus objetivos, la educación del talento académico de los estudiantes desde

\footnotetext{
${ }^{1}$ Profesor Auxiliar

${ }^{2}$ Profesora Auxiliar

${ }^{3}$ Profesor Auxiliar
} 
la Física. Se presentan herramientas al docente para su preparación en la educación del talento académico de los estudiantes desde la Física, a partir de nuevos fundamentos semióticos que permiten utilizar los códigos culturales y cotidianos para motivar intrínsecamente a los estudiantes, se proponen principios, definiciones en que esta se sustenta, así como sus elementos condicionantes, dimensiones e indicadores, que se concretan a través de las orientaciones con pasos metodológicos.

Palabras claves: Educación, Talento académico, Física, preparación docente.

\section{Introducción.}

Uno de los objetivos más importantes de la educación es favorecer las potencialidades de todos los estudiantes para que alcancen el máximo desarrollo académico y personal a lo largo de su escolarización, para lo cual hay que crear las condiciones necesarias desde la enseñanza. Es justamente desde esta perspectiva que la educación del talento en los estudiantes cobra especial relevancia.

La educación del talento de los estudiantes es, en ocasiones, poco atendida por el sistema educativo, porque se brinda poca ayuda diferenciada. Al mismo tiempo, prevalece la interpretación parcial y sesgada de la noción de estudiantes superiores, entendiéndola solo en el caso de los sujetos, que por sus especiales circunstancias están en desventaja y tienen mayores dificultades para beneficiarse de la educación escolar.

Sin embargo, contrariamente a lo que muchas veces se piensa, no resulta fácil organizar un sistema educativo ajustado a sus necesidades de los estudiantes talentos, además, ellos en gran medida, no han sentido la actividad de estudios como algo gratificante y con sentido para la vida.

Debe reflejarse en la estrategia de trabajo educativo de los centros, la orientación ajustada a las necesidades del talento de los estudiantes que ha de ser planificada, desarrollada y evaluada con rigor. Esta es una actividad que precisa y genera procesos de colaboración entre los profesores, los estudiantes, la institución y las familias.

La comunidad científica internacional cada vez presta mayor atención a esta problemática. Dentro de los autores consultados, en el plano internacional, están: Rubinstein (1972) destacó la relación entre el genio y el talento; De Bono (1974) el cual propone procedimientos para estimular la creatividad; Renzulli (1992), propone un modelo cognitivo del talento; Silverman (1993) y Aleçar y Spoza (2001), tipifican las diferencias interindividuales en cuanto a estilos cognitivos y de aprendizaje; Gagné (2007) hace una propuesta cognitiva centrada en conocimientos y destrezas; Aroça (1974) plantea que para obtener éxito es necesario un alto esfuerzo volitivo y utilizar sus habilidades; Tannebaum (1993); y Mönks y Van Boxtel (2003) amplían el modelo de Renzulli incluyendo el contexto social. 
En Cuba, en los últimos años del siglo XX, se han llevado a cabo diversas investigaciones orientadas a fundamentar e instrumentar alternativas para atender el talento y la creatividad de los estudiantes (Lorenzo, 1996; Córdova, 1996); Castro, 1997; Amechazurra, 1999; Ávila, 1999); Gallardo, 2000; Guerra, 2001; Martínez, 2001; Pérez, 2002; Castellanos, 2003; Campos, 2007; López, 2008; Delgado, 2011; Lorenzo, 2012). Estas investigaciones están dirigidas, fundamentalmente, a la enseñanza primaria y secundaria; mientras que en el preuniversitario solo se trabaja la preparación de estudiantes, que participan en los concursos de conocimientos.

En la provincia de Mayabeque, se constató que todo lo relacionado con la atención al talento académico en los estudiantes se concretaba, fundamentalmente, en actividades de preparación para concurso nacionales e internacionales, además, los estudiantes estaban poco motivados por el estudio de las ciencias exactas.

En el caso de la asignatura Física, la cantidad de estudiante que se acercaba a la preparación oscilaban entre tres y cuatro, muchos de ellos no ingresaban a las carreras universitarias en esta especialidad. Sin embargo, existían otros estudiantes que mostraban interés por la Física, aun cuando no eran etiquetados como talento académico en esta asignatura. A partir de esta situación, es que se considera desarrollar una investigación dirigida a la educación del talento académico en los estudiantes desde la Física.

Se comprobaron dificultades en la atención al talento académico en los estudiantes, tales como: los componentes del proceso enseñanza-aprendizaje de la Física (objetivos, contenidos, métodos y evaluación) poseen poca correspondencia con el nivel de la educación del talento académico de los estudiantes; los profesores realizan insuficientes acciones educativas encaminadas al desarrollo del talento académico en los estudiantes desde la Física; organizan su trabajo con los estudiantes de manera espontánea y no sistemáticamente; dentro de la atención existen limitaciones en el protagonismo de los estudiantes y un número insuficiente de actividades de consolidación de sus resultados; la identificación del talento académico se realiza, según sus resultados en pruebas sobre contenidos precedentes; y la familia tiene insuficiente preparación para la atención de la educación del talento académico en los estudiantes desde el hogar.

Se plantea como objetivo del presente artículo: Proponer orientaciones teóricas metodológicas para la preparación del docente relativo a la educación del talento académico de los estudiantes desde la Física en la provincia de Mayabeque.

\section{Desarrollo.}

La educación implica el desarrollo de las dimensiones del pensar, sentir y actuar, en todas las áreas de relación social con la persona, abarca los procesos de instrucción y formación integral de los sujetos en valores (D'Angelo, 2004). Esta proporciona un sistema de influencias materializado por la cultura. Permite modelar y favorecer el desarrollo del sujeto, 
porque admite penetrar en su entendimiento, explicación y potenciación del desarrollo humano.

La educación, constituye un sistema complejo de influencias, en las que participa toda la sociedad. Estas influencias, que se ejercen con el objetivo de asegurar la asimilación y reproducción de toda la herencia cultural anterior, así como de las relaciones sociales existentes. Por regla general, actúan como procesos de cooperación y comunicación social, en que los hombres desempeñan el papel de sujetos activos y creadores.

La educación constituye el medio fundamental para la socialización del sujeto, a través de ella la sociedad logra la asimilación y objetivación, en cada individuo, de los contenidos socialmente válidos, expresados en los sistemas de normas y valores aceptados por la misma sociedad y la socialización abarca todos los aspectos de la vida de la sociedad.

En la actualidad, son necesarios estos conocimientos para contribuir al enriquecimiento de la pedagogía profundizando en las regularidades sicológicas del comportamiento humano, el sistema de relaciones sociales que lo determina, la manera en que ocurren las mediaciones y las relaciones comunicativas. La pedagogía como ciencia que se ocupa de la transformación del ser humano, debe dialogar de forma interdisciplinaria y transdisciplinaria con otras ciencias que tributen a este objeto a través de diferentes aristas de este para favorecer las necesidades crecientes del desarrollo actual.

Al realizar una definición operativa sobre educación del talento, resulta difícil el estudio epistemológico; porque en la bibliografía científica se emplean categorías como superdotado, genio, precocidad, creatividad e inteligencia, incluso, a veces uno sirve de sinónimo o forma parte de la definición del otro, por tal razón es difícil definir la educación del talento sin un análisis de cada categoría por separado.

A partir de la sistematización de los autores nombrados y algunos más, se sistematizan las principales regularidades operativas en función de esta investigación. Luego, se propone la siguiente definición sobre la educación del talento académico:

Educación del talento académico: Proceso complejo que se dirige con motivación, animación y estimulación de las potencialidades humanas, conduce a la inteligencia y creatividad hacia un objetivo dado y orienta el pensamiento y la conducta del estudiante, teniendo en cuenta el contexto sociocultural, sus relaciones cotidianas dentro del mismo y su implicación mediadora en este proceso.

La educación del talento académico contempla varias dimensiones: motivacional afectiva y volitiva de la personalidad; inteligencia académica; creatividad; contexto sociocultural y la mediación pedagógica.

- Motivacional afectiva y volitiva del estudiante hacia la educación del talento académico desde la Física: Dentro de esta dimensión, se tiene en cuenta la 
motivación intrínseca o extrínseca del estudiante que es observable a través de su implicación con la tarea académica desde la Física. Se considera también la voluntad concretada en la perseverancia del estudiante, y los valores y sentimientos son medibles a partir del compromiso social del estudiante con su contexto cultural.

- Inteligencia académica desde la Física: Esta dimensión se concreta en varios indicadores observables y medibles: capacidad para discernir patrones lógicos o numéricos en la resolución de problemas de Física, capacidad para manejar cadenas extensas de razonamiento en la resolución de problemas de Física, capacidad para percibir el mundo espacial visual y realizar transformaciones en las propias percepciones iniciales.

- Creatividad desde la Física: La creatividad es observable a través de los siguientes indicadores: flexibilidad, originalidad, capacidad para identificar problemas, imaginación, curiosidad, toma de riesgos, tolerancia a la ambigüedad, alto nivel de energía, intuición e independencia en la resolución de tareas docentes de Física.

- Contexto sociocultural: El contexto sociocultural es complejo porque lo integran diferentes indicadores los coetáneos se evalúa a partir de la opinión que tienen sobre el talento académico en los estudiantes ; en los profesores se evaluará la preparación para educación del talento académico en los estudiantes ; los padres y familiares, instituciones estatales y las redes sociales, se evalúa su contribución para la educación del talento académico en los estudiantes desde la Física.

- Mediación pedagógica: Esta dimensión se divide en dos indicadores, uno la mediación sicopedagógica que se concreta en: diagnóstico dinámico para la identificación del talento académico, niveles de ayuda al talento académico para su estimulación, actividades lúdicas, complejidad de la actividad. El otro resulta la mediación cultural: es observable a partir del uso de la Física como metalenguaje, uso de la Física como texto cultural, uso del método científico experimental y proyectos problémicos de la realidad.

Para orientar a los profesores de tal manera, que puedan concretar estas dimensiones en el mejoramiento de la educación del talento académico, se proponen las siguientes orientaciones metodológicas:

Se recomienda metodológicamente no partir de la Física como contenido que problematiza la práctica de forma abstracta y que tradicionalmente se hace. Se indica diagnosticar e identificar dentro de la cultura discursiva del estudiante algunas áreas del conocimiento que resultan para él, parte de su vida cotidiana y contextual, como por ejemplo el uso del celular, la fotografía, el audiovisual, la cosmología y la agricultura, el uso de los deshechos de producción y recursos naturales. Estas áreas requieren de estudios inter y transdisciplinario, pero la Física tiene un alto componente en su desarrollo.

Se sugiere crear proyectos científicos con los estudiantes, que trabajen con la vía inductiva de la realidad a la explicación científica o cultural, con la utilización como argumento la 
Física, para llegar a los conocimientos y habilidades como el movimiento, la localización, ondas electromagnéticas, imagen, óptica, color, efecto fotoeléctrico, astronomía, energías y gases.

Y luego de tener estos conocimientos emplear la vía deductiva, esto permitirá buscar fundamentos interdisciplinares que admitan la explicación del problema científico analizado; se acepta y se estimula la vía abductiva, la que conlleva una solución sorprendente del problema.

Se ofrecen como alternativa los siguientes pasos metodológicos para la educación del talento académico en los estudiantes:

1. Búsqueda de problemas del contexto cotidiano (tablas, celular, agricultura, vialidad)

2. Socialización de los problemas e identificación de un grupo de ellos relacionados con los contenidos del programa de Física

3. Análisis teórico y práctico de los problemas a partir de la deducción, inducción y abducción

4. Búsqueda bibliográfica, consulta en internet y a expertos de los contenidos de Física para su resolución

5. Identificar y socializar estrategias utilizadas para la resolución de problemas.

6. Comprobación de la resolución del problema

7. Evaluación, coevaluación y heteroevaluación

A estas orientaciones metodológicas, se le agregan algunas definiciones operativas que permiten entender y operacionalizar mejor las orientaciones recibidas:

- Contexto sociocultural: Es una configuración de diferentes componentes sociales: familia, coetáneos, redes sociales, instituciones con las que se identifican, que interactúan de forma directa y cotidiana con los estudiantes.

- Motivación intrínseca: Son configuraciones subjetivas inherentes al sujeto que le produce altos niveles de satisfacción y fuerza por lo que se hace que disfrute así de la actividad con mejores niveles de desempeño.

- Motivación extrínseca: Son configuraciones subjetivas estimuladas desde el exterior a través de diferentes medios que organizan y orientan la actividad del estudiante centrado en una meta. 
- Mediación pedagógica: Es un sistema estratégico de actividades que le facilita al profesor la mediación entre el contexto sociocultural y la experiencia del estudiante y el aprendizaje de la Física a través de dos procesos, la mediación psicopedagógica y la mediación cultural.

- Mediación psicopedagógica: Espacio de interacción donde el profesor define y determina las acciones psicopedagógicas a concretar, a partir del diagnóstico dinámico del talento actual y de doble excepcionalidad quien determina los niveles de ayuda (motivación, orientación, estimulación), para desarrollar el talento potencial de todos hacia el nivel del talento actual, como proceso dialéctico y sistemático.

- Mediación cultural: Es la utilización de significados y tradiciones cotidianas desde áreas del conocimiento transdisciplinares, para la solución de problemas científicos empleando la vía inductiva, al aplicar conocimientos y habilidades de la física y la vía deductiva, para la resolución de problemas y en la relación dialógica con los componentes sociales de la cultura, se utiliza la vía abductiva para dar soluciones creativas.

- La Física como texto cultural y metalenguaje: La física es un metalenguaje cuyos códigos constituyen la estructura de un producto cultural que redefine sistemáticamente los fenómenos de la naturaleza y sus relaciones, esto la convierte en un texto que entra en interacción permanente con sus interpretantes y el objeto de la naturaleza a la cual ella hace referencia.

Y para implementar la educación del talento académico, se recomiendan las siguientes premisas:

Uso de recursos y medios que permitan la formación conceptual metodológica del estudiante.

Para que el estudiante desempeñe el papel de sujeto de su propia formación, tiene necesariamente que aprender "a hacer", a "ser" y a "convivir" con los demás. Esto se logra al operar directamente con los diferentes objetos, procesos y leyes, lo cual se favorece mediante el uso de medios y recursos de diferentes tipos.

En el transcurso de la presente investigación, se diseñó y elaboró por parte del autor un conjunto de instrumentos para el estudio de fenómenos físicos para la realización de experimentos en clases constituyen componentes esenciales del proceso de enseñanzaaprendizaje, para el estudio integral del fenómeno físico mediante la combinación con otros medios y recursos. 
Se utiliza un software, que ha sido tomado de la red y adaptado en el presente estudio, para ser usado sin necesidad de conexión a Internet, este hecho permite al estudiante desde cualquier computadora usarlo para estudiar la materia.

\section{Atención a la diversidad para favorecer la formación integral.}

La diversidad educativa en el aula se expresa a través de la diversidad tanto de las características propias de cada estudiante (cognitiva, afectiva, motivacional y psicosocial), la socioeconómica y la cultural. A partir de aquí es comprensible que en cada grupo existe una gran variedad en las aulas, lo que por supuesto conlleva a infinidades de demandas educativas y a la necesidad de plantear estrategias que den respuestas a las mismas. En la atención a la diversidad, se materializa la unidad de lo individual y lo social como fuente motriz del desarrollo del estudiante.

En el caso de la presente propuesta, y como vía de materializar una de las regularidades señaladas se sugiere como vía fundamental la organización en pequeños grupos a la hora de realizar los experimentos y de trabajar con el software, la atención a las diferencias individuales es posible a la hora de determinar los integrantes de los pequeños grupos, al brindarle una atención diferenciada a los que presentan dificultades en la solución de los problemas, en el uso de la computadora, no solo por el docente sino también por los estudiantes del mismo grupo. Por otra parte, cumplir esta premisa significa tomar en cuenta los intereses de los estudiantes para la realización de las tareas docentes.

Lo expresado permite aplicar un enfoque diferenciado y si el estudiante prefiere usar el software primero y después realizar el experimento, o si desea hacerlo de manera inversa puede hacerlo.

\section{Enfoque problematizador del contenido para lograr una lógica interpretativa integradora.}

Los estudiantes aprenden mejor cuando se enfrentan a situaciones que les hacen reflexionar, cuestionar. La lógica gnoseológica de la Física permite problematizar el contenido, planteando tareas, problemas, ejemplificando, basado en contradicciones. También la experimentación y la demostración posibilitan enfrentar a los estudiantes con problemas de diferentes índoles, que despierten la curiosidad y el interés por aprender. En la presente propuesta la problematización del contenido se cumple en cada momento del desarrollo del proceso siempre que se creen las condiciones para ello, por cuanto se puede partir de una situación problémicas y de allí pasar a la observación, los problemas pueden ser planteados por el profesor o por parte de los estudiantes cuando trabajan con el software o cuando realizan el experimento. Cumplir con este requerimiento posibilita el desarrollo de la capacidad interpretativa porque el estudiante debe interpretar, explicar, argumentar, además de potenciar la formación conceptual metodológica. 


\section{Clima que favorezca la formación de los estudiantes.}

Un adecuado clima relacional, activo y positivo, de cooperación y participación, donde los errores y aciertos sean fuentes para el aprendizaje y los estudiantes puedan disfrutar del propio proceso, es un requisito fundamental, lo contrario sería un clima tenso, cargado de autoritarismo por parte del profesor donde predomine su participación en detrimento de la autoactividad del estudiante.

Al sustentarse la presente investigación en los postulados de Vigotsky, se considera la mediación de otros sujetos en el aprendizaje personal, en un contexto dialógico y colaborativo, mediante el cual los estudiantes participen activamente de la cultura. Disentir, valorar, validar, consensuar son aspectos no solo de la construcción intersubjetiva del conocimiento, sino también de los modos de pensar y conducirse. De este modo, se contribuye a la formación cultural de los estudiantes como una consecuencia de la utilización de las potencialidades de la Ciencia Física y las potencialidades de las Ciencia Didáctica.

\section{Consideración de las preconcepciones del estudiante como vía para lograr la unidad del significado y el sentido.}

Aún en la actualidad, muchos profesores desarrollan su actividad docente como si la mente de los estudiantes fuera un recipiente vacío en el que se colocan los conocimientos, de esta manera el aprendizaje se concebía como un proceso de adquisición de información, en primer orden y solo en segundo lugar como un proceso de desarrollo de habilidades y capacidades.

Es conocido que los estudiantes poseen un conjunto diverso de ideas previas o preconcepciones sobre los contenidos científicos que casi siempre son erróneas. La solución de las contradicciones entre las ideas previas o preconcepción de los estudiantes y las ideas científicamente probadas constituye una fuerte base metodológica para el proceso de enseñanza-aprendizaje de la Física. La vinculación de la teoría con la práctica permite la creación de espacios para lograr la unidad de significados y sentidos. Se adquiere un conocimiento científico lo que se relaciona con un significado verdadero sin sesgos y el estudiante incorpora ese significado a la solución de tareas que pueden tener una amplia aplicación.

El diagnóstico realizado muestra que los estudiantes poseen muchas preconcepciones relacionadas con la óptica. En la medida que el profesor identifique las preconcepciones de los estudiantes puede dirigir conscientemente las acciones que permiten eliminarlas y de esta manera se vincula la Física con la vida.

Vinculación del contenido con el contexto y la situación actual como vía para lograr la unidad de la formación conceptual metodológica y la formación cultural.

Es de vital importancia que en el proceso de enseñanza-aprendizaje se desarrolle en los estudiantes la capacidad de resolver problemas que expresan una realidad cotidiana, de 
otorgar significados a lo que se aprende en correspondencia con las condiciones actuales del desarrollo social y tecnológico, de aprender a adaptarse a situaciones nuevas y de sentirse responsables con la transformación de la realidad.

Tomar en cuenta esta condición significa el desarrollo de la capacidad mediante el estudio de leyes y fenómenos físicos de poder explicar la realidad circundante, de familiarizarse con la relación causa-efecto y su amplia significación para el análisis de cualquier fenómeno no solo físico sino también social.

La observación, la experimentación y otros métodos propios de esta ciencia ayudan al estudiante a la comprensión del entorno, implica fijar la atención, discriminar elementos, relacionarlos, interpretarlos. Por esta razón, se hace énfasis en la presente propuesta en el planteamiento de problemas, de tareas contextualizadas para que, además, de potenciar el significado social del contenido, también se eleve la motivación hacia las clases.

\section{Conclusiones.}

- Las orientaciones que ofrecen al profesor de Física para trabajar la educación del talento académico emergen de la interrelación dinámica de los subsistemas formación conceptual metodológica y la formación cultural, en una síntesis que articula la Ciencia Física y la Didáctica que tiende a favorecer un proceso formativo trascendente.

- Se orienta cómo realizar un proceso formativo de construcción del conocimiento y el desarrollo de otras potencialidades del estudiante en las diferentes esferas del pensar, el sentir y el actuar, con un abordaje del proceso de enseñanza aprendizaje de la Física, que se dinamice por la creación de espacios para la construcción de significados y sentidos, a partir de los discursos cotidianos de los estudiantes, donde descubran las características gnoseológicas de la Física y sus posibilidades de influencia en el proceso formativo y resolución de problemas del contexto cultural.

\section{Referencias bibliográficas.}

Alegría, I., Lay, S.-1., Calderón, C., \& Cárdenas, M. (2010). El proceso de construcción y validación de la Escala de Identificación de Talento Académico "EDITA". Estudios Pedagógicos, XXXVI (2), 25-39.

Alonso, A., Cairo, E. y Rojas, R. (2007). Psicodiagnóstico. Selección de Lecturas. Ciudad de La Habana, Cuba: Félix Varela.

Atenberg, J. R. (2005). 3.4. Cultura e inteligencia. En Z. Bello, \& N. Estévez, Selección de Lecturas de inteligencia humana (pp. 345-413). Ciudad de La Habana, Cuba: Félix Varela. 
Bedia, L. (2010). La superdotación y el talento: una aproximación a su desarrollo conceptual. Recuperado el 9 de enero de 2012, de Monografía.com: htpp//:www.monografias.com

Campos, E. (2006). Estrategia metodológica para la preparación de alumnos que participan en los concursos de español-literatura en preuniversitario. (Tesis de Doctorado). Santa Clara, Cuba: Universidad Central de las Villas "Marta Abreu”.

Castellanos, D. (2014). Talento: estrategia para su desarrollo. La Habana,Cuba: Pueblo y Educación.

Castro Díaz-Balart, F. (2002). Cuba. Amanecer del Tercer Milenio. Ciencia, Sociedad y Tecnología (compilación). Barcelona: Debate.

Correa, C. (2006). Propuesta metodológica para diagnosticar inteligencias múltiples en estudiantes universitarios. (Tesis de Doctorado). Cienfuegos, Cuba: CEDDES.

Crawford, R. P. (1954). Techniques of creative thinking. New York: Hawtorn Books. talent. En R. Sternberg, \& J. E. Davinson, Conceptions of giftedness. Cambridge: Cambridge University Press.

D'Angelo, O. (2001). Sociedad y educación para el desarrollo humano. Ciudad de La Habana, Cuba: Editorial Pueblo y Educación.

De Bono, E. (1974). El pensamiento lateral. Manual de creatividad. Barcelona: Paidos.

Domínguez, L. (2007). Psicología del desarrollo. Problemas, principios y categorías. Ciudad de La Habana, Cuba: Félix Varela.

Fariñas, G. (2006). Psicología y educación de la creatividad. En Fariñas, G. (2006) Psicología, educación y sociedad (p. 34). Ciudad de La Habana, Cuba: Félix Varela.

Gardner, H. (1995). Inteligencias Múltiples. La teoría en la práctica. Barcelona, España: Editorial Paidos.

Heller, K.A. Y Viek, P. (1999). Support for university students: Individual and social factors. En: Grayson, P. (Ed). (1999). The performance of gifted high school students in university. Toronto: Institute for Social Research, New York University.

Lorenzo, R. (2013). Talento, creatividad, empresa. La Habana: Academia.

Lorenzo, R. y Martínez, M. (1999). Talento para la ciencia: estrategia para su desarrollo. La Habana: Academia.

Marina, J. A. (2015). El talento de los adolescentes. (3ra ed.). España: Editorial Ariel. 
Mönks, F. (1992). Development of Giftedness a life-span perspective. En K. A. Heller y E. A. Hany (Eds.) (1992). Competence and Responsibility (Vol. 2) (pp.141-146). Munich: Hogrefe \& Huber Publishers.

Vera, C. (2001). Manual para la identificación de talentos escolares en la Educación Primaria. (Tesis en opción al Título Académico de Máster en Psicología Educativa). Ciudad de La Habana, Cuba: Facultad de Psicología, Universidad de La Habana.

Vera, C. y Vera, N. (2003). Atención educativa a la diversidad de estudiantes talentosos en la escuela primaria. En Castellanos, D. (Comp.) (2003). Talento: Estrategias para su Desarrollo. Ciudad de La Habana, Cuba: Editorial Pueblo y Educación.

Vigotsky, L.S. (1982). Pensamiento y Lenguaje. Ciudad de La Habana, Cuba: Editorial Pueblo y Educación.

Vigotsky, L.S. (1987). Historia del desarrollo de las funciones psíquicas superiores. Ciudad La Habana, Cuba: Editorial Científico-Técnica. 


\section{Para citar el artículo indexado}

León Arencibia, A., Dorvigny González, B. de la C., \& Rodríguez Brito, B. E. (2020). La educación del talento académico en los estudiantes desde la física. AlfaPublicaciones, 2(2), 47-59. https://doi.org/10.33262/ap.v2i2.28

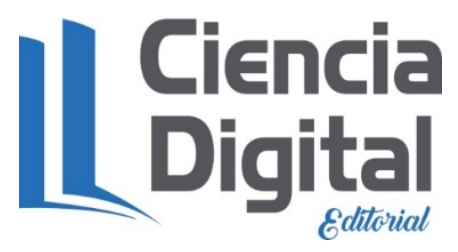

El artículo que se publica es de exclusiva responsabilidad de los autores y no necesariamente reflejan el pensamiento de la Revista Alpha Publicaciones.

El artículo queda en propiedad de la revista y, por tanto, su publicación parcial y/o total en otro medio tiene que ser autorizado por el director de la Revista Alpha Publicaciones.
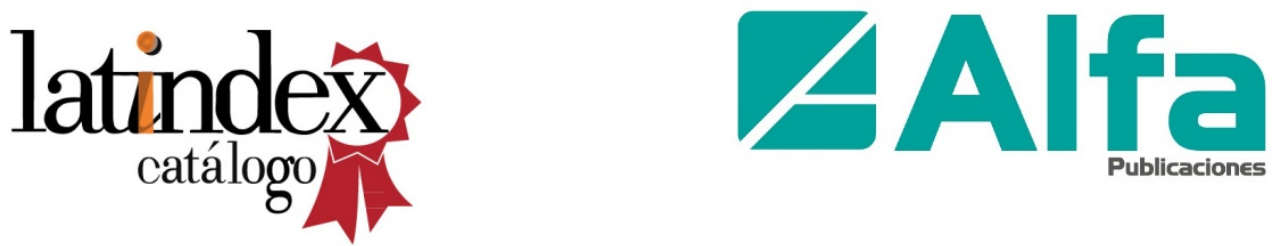